\title{
Silver-Russell syndrome
}

INSERM

\section{Source}

INSERM. (1999). Orphanet: an online rare disease and orphan drug data base. SilverRussell syndrome. ORPHA:813

Silver-Russell syndrome is characterized by growth retardation with antenatal onset, characteristic facies and limb asymmetry. 